\title{
SURFACE MICROMACHINED DIAPHRAGM PRESSURE SENSORS WITH OPTIMIZED PIEZORESISTIVE SENSING RESISTORS
}

\author{
Liwei Lin, Weijie Yun*, Huey-Chi Chu and Mu Chiao \\ Institute of Applied Mechanics, \\ National Taiwan University, Taipei, Taiwan \\ *BEI Electronics Inc., California, USA
}

\begin{abstract}
Surface micromachined diaphragm pressure sensors utilizing " $n$ " shape piezoresistive sensing resistors have been successfully demonstrated. These micro pressure sensors were fabricated by an integrated process combining both the surface and bulk micromachining techniques. A newly developed design optimization principle was used for the design of the piezoresistive sensing resistors. An absolute type pressure sensor with 100 Psi full scale output was built based on the optimal design principle as well as the integrated micromachining process. Experimental results showed that sensitivity of $0.15 \mathrm{mV} / \mathrm{V} / \mathrm{Psi}$, linearity error of $\pm 0.1 \%$ FSS (Full Scale Span) and pressure hysteresis of $0.02 \%$ FSS has been achieved.
\end{abstract}

\section{INTRODUCTION}

Micro pressure sensor is one of the most successful products fabricated by micromachining technology. For years, bulk micromachined diaphragm pressure sensors have been widely used in industry. Recent development in surface micromachining technologies has successfully demonstrated that micro diaphragms made by surface micromachining can be used to make pressure sensors. For example, the absolute type pressure sensors which require vacuum cavities underneath diaphragms made by surface micromachining have been made by a reactive gas sealing process [1] or an LPCVD (Low Pressure Chemical Vapor Deposition) sealing process [2]. On the other hand, the differential type pressure sensors which need access channels from both sides of the surface micromachined diaphragms have been fabricated by a back side silicon etching process [3]. These surface micromachined diaphragm pressure sensors may have advantages over bulk micromachined types for the following reasons: larger devices/area ratio, easier control of the diaphragm thickness, better integration compatibility with IC processes and no silicon to glass bonding for the diaphragm sealing process [4].
Papers in the literature have already addressed the process [5] and design [6] issues for the bulk micromachined diaphragm pressure sensors while that for the surface micromachined types need further investigation. It is desirable to explore more fabrication techniques and design schemes in order to fabricate highly sensitive and reliable surface micromachined diaphragm pressure sensors. In this paper, we present an integrated process and an optimal design concept of sensing resistors for surface micromachined diaphragm pressure sensors.

\section{FABRICATION PROCESSES}

Figure 1 is the schematic diagram of a surface micromachined diaphragm with a vacuum cavity underneath. The square shape diaphragm is $100 \mu \mathrm{m}$ long and only half of the device is drawn in order to illustrate the vacuum cavity and possible positions for the sensing resistors. When an outside pressure is applied to the top surface and deflects the diaphragm, the resistance of the piezoresistive sensing resistor changes.

In order to create the vacuum cavity, LPCVD polysilicon was used to hermetically seal the large cavity. This process was adopted from a similar sealing process previously used for vacuum encapsulated lateral micro resonators [7]. It creates the diaphragm and at the same

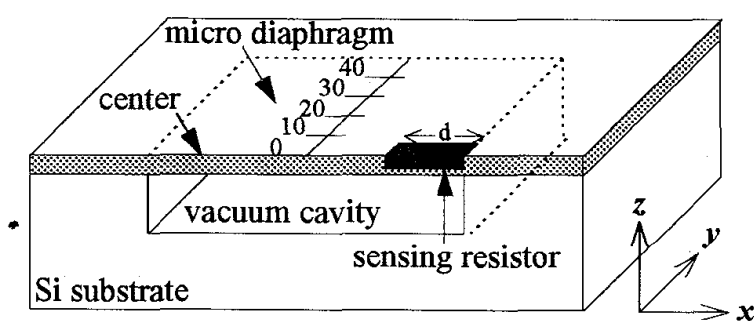

Fig. 1 Schematic diagram of a surface micromachined diaphragm with a vacuum cavity underneath (only half sensor is shown). 


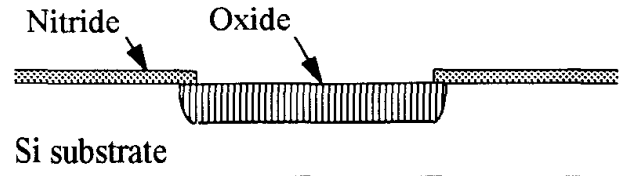

(a)
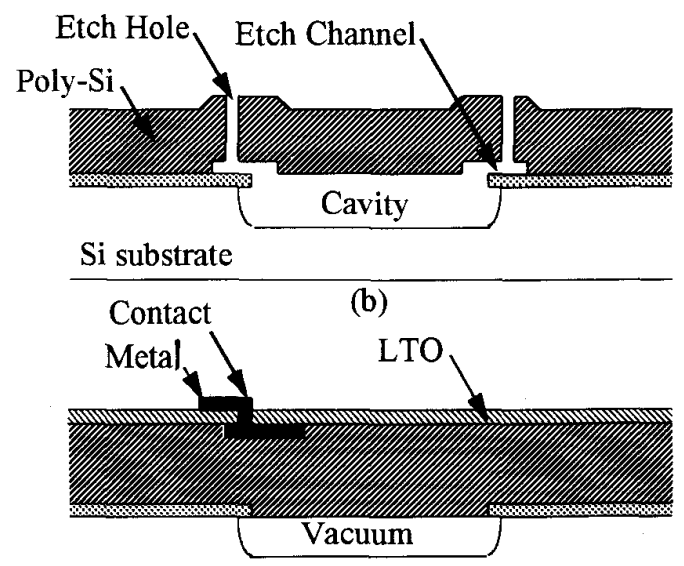

Si substrate

(c)

Fig. 2 Process sequences of surface micromachined diaphragm pressure sensors. (a) after cavity oxidation, (b) after LPCVD vacuum encapsulation of the cavity, (c) final cross section.

time seals the cavity in vacuum environment. Since the low vacuum cavity is built below the surface micromachined diaphragm via the LPCVD deposition process, no extra post vacuum packaging step is required.

To build such a pressure sensor by surface micromachined diaphragm, a series of micromachining process are performed. First, wafers are cleaned and deposited with a thin layer of LPCVD silicon nitride and the cavity area is defined by a first mask via dry etching process to create a cavity about $1 \mu \mathrm{m}$ deep. The wafer is then going through thermal oxidation process to create about $2 \mu \mathrm{m}$ thick silicon dioxide at the cavity area as illustrated in Fig. 2(a). LPCVD oxide of $0.5 \mu \mathrm{m}$ thick is then deposited and etch channels are defined by dry etching with the second mask. It is followed by a $1 \mu \mathrm{m}$ LPCVD polysilicon deposition, etch holes patterning and dry etching by using the third mask. The oxide in the etch channels and the cavity are now cleared by concentrated $\mathrm{HF}$ etching and the cross sectional view at the end of this step is shown in Fig. 2(b). The second layer of polysilicon is then deposited by a second LPCVD polysilicon deposition which seals the etch holes and channels creating a vacuum environment inside the cavity. The piezoresistive resistors are then implanted by using the fourth mask on top of the diaphragm to create piezoresistive resistors. An LPCVD oxide passivation layer is deposited and standard contact hole opening

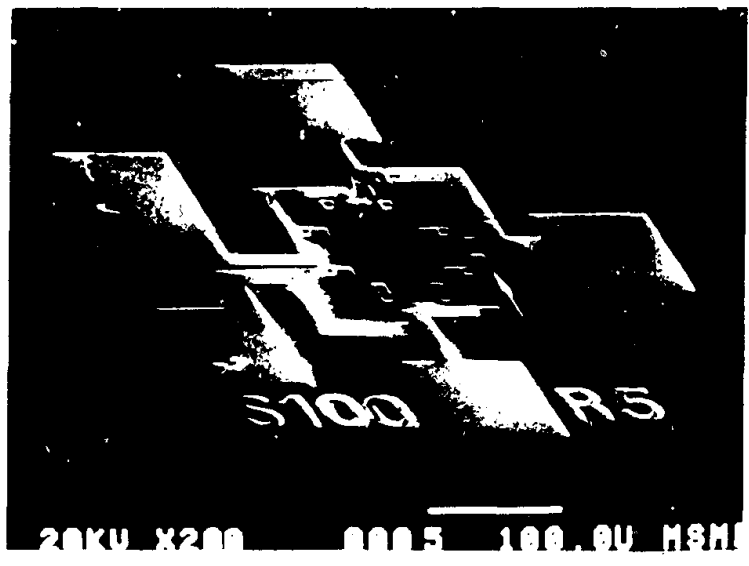

Fig. 3 An SEM micrograph of a $100 \mu \mathrm{m}$ wide square diaphragm surrounded by eight etch channels.

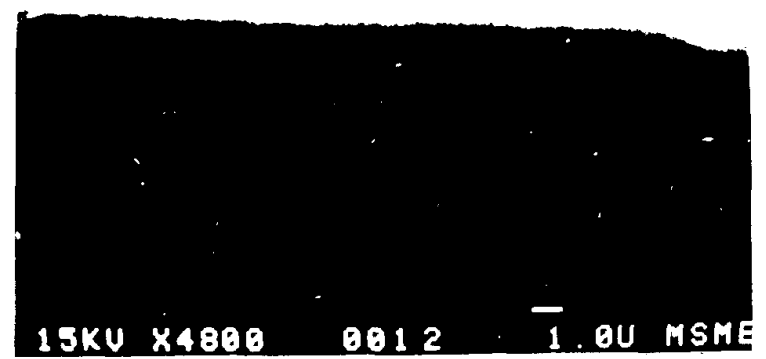

Fig. 4 An SEM micrograph of a cleaved device with cavity. The depth of the cavity is about $2 \mu \mathrm{m}$.

(Mask \#5) and metallization (Mask \#6) are followed. The final cross section is seen in Fig. 2 (c).

Figure 3 is a SEM micrograph of a fabricated absolute type pressure sensor which has a $100 \mu \mathrm{m}$ wide square shape diaphragm. The eight etch channels around the diaphragm can be clearly identified. They are used as access channels to remove the sacrificial thermal oxide before the vacuum encapsulation of the micro diaphragm. Figure 4 shows the SEM micrograph of a cleaved device with the cross section of the cavity clearly seen. The depth of the cavity is about $2 \mu \mathrm{m}$ and the thickness of the diaphragm is about $2 \mu \mathrm{m}$ as shown.

\section{SENSING RESISTOR OPTIMIZATION}

Besides the fabrication processes, design optimization for the built-in piezoresistive sensing resistors is another essential issue to improve the performance of a pressure sensor. A complete analytical model has been developed to calculate the performance of the individual sensing resistors on the surface 


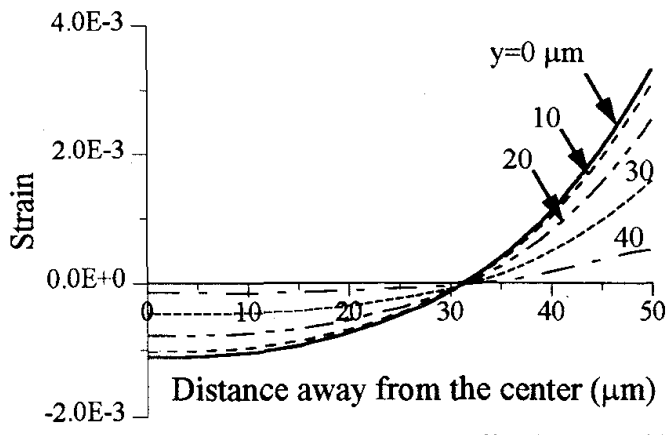

Fig. 5 The $\mathrm{x}$-directional strain distribution on different y positions of $0,10,20,30$ and $40 \mu \mathrm{m}$, respectively.
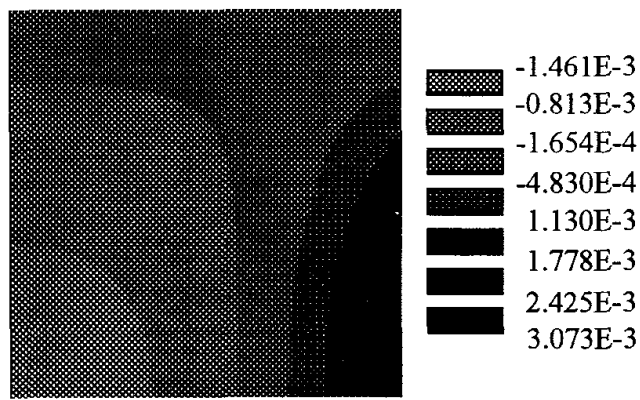

Fig. 6 The $x$-directional strain distribution on the top right quarter of the diaphragm calculated by finite element analysis.

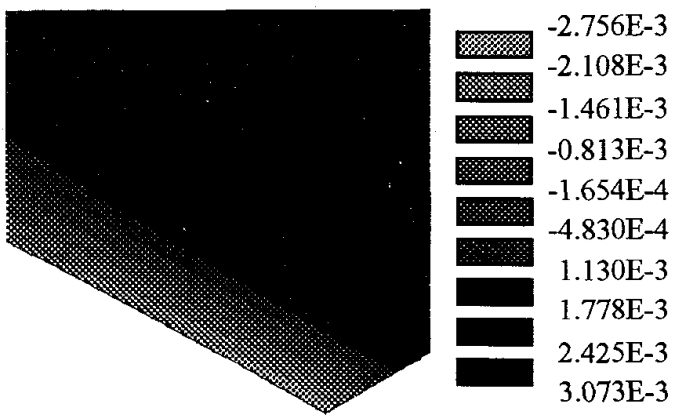

Fig. 7 The $x$-directional strain distribution calculated by three dimensional finite element analysis.

micromachined diaphragms. The best sensing resistor shape and position are then simulated by finite element analysis to achieve design optimization.

As seen in Fig. 1, a possible sensing resistor with length " $d$ " is placed at the edge of the center line $(y=0)$. There are many possible positions along the $y$ direction for the placement of the resistor. The resistors which are placed in parallel to the $y$ direction will be sensitive to the $x$-directional strain. The $x$-directional strain of the square shape diaphragm can be calculated theoretically by the method developed by S.Timoshenko [8].

These $\mathrm{x}$-directional strains along the $\mathrm{y}$ direction have been calculated as shown in Fig. 5. These data were calculated for a $100 \mu \mathrm{m}$ square shape polysilicon diaphragm with $2 \mu \mathrm{m}$ in thickness and under 100 Psi

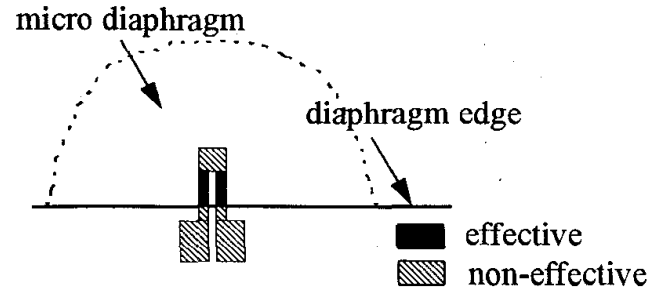

Fig. 8 The " $n$ " shape resistor.

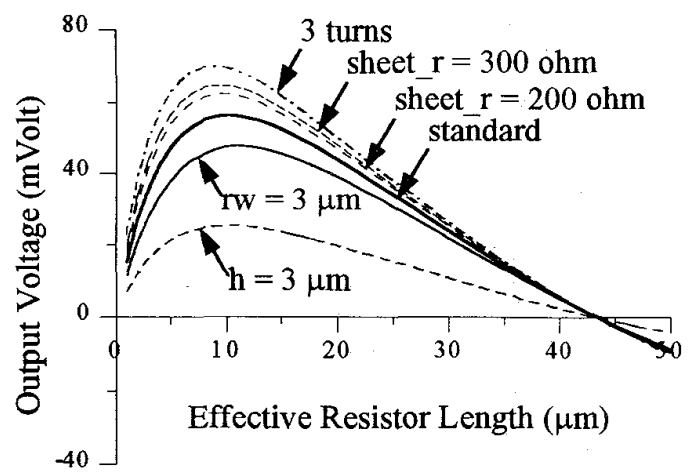

Fig. 9 Effective resistor length vs. output voltage with varying sheet resistance (sheet_r), resistor width (rw), resistor turns and diaphragm thickness $(\mathrm{h})$.

pressure. It is clear that the sensing element should be placed as close as to the center line $(y=0)$ and at the edge of the diaphragm for maximum $\mathrm{x}$-directional strain. Consistent result is found by using a two-dimensional ANSYS finite element analysis [9] as seen in Fig. 6, where only the right top quarter of the diaphragm is simulated. The three-dimensional ANSYS simulation around the bottom right corner of Fig. 6 is shown in Fig. 7. It reveals that the maximum strain occurs not only near the edge but also at the top surface of the diaphragm. In fact, the bottom side of the diaphragm will be in compression while the strain changes from tensile state at the top to compressive state at the bottom. Hence, for high sensitivity, the implanted resistor should be as shallow as possible and the passivation LTO layer should be as thin as possible.

According to the above analysis, an " $n$ " shape resistor placed at the center edge of the diaphragm has been used for our device as shown in Fig. 8. As suggested in Fig. 5, the length of the resistor also determines the sensor sensitivity. To determine the optimal resistor design, the effect of resistor length on sensor output is simulated with the results presented in Fig. 9. In our prototype sensor design, only two " $n$ " shape resistor are active in the Wheastone bridge while the other two are reference resistors. Moreover, the turning portion of the resistor is treated as non-effective. A 100 Psi pressure is used for the simulation on a $100 \mu \mathrm{m}$ wide square shape membrane with $2 \mu \mathrm{m}$ in thickness. The standard resistor is a one turn or one " $n$ " shape type, with sheet resistance 
of $100 \mathrm{ohm}$, width of $2 \mu \mathrm{m}$ and non-effective resistor length of $2 \mu \mathrm{m}$. The best resistor length is about $10 \mu \mathrm{m}$ for a standard type resistor as observed in Fig. 9. Increasing the sheet resistance (sheet_r) from $100 \mathrm{ohm}$ of the standard type to 200 or $300 \mathrm{ohm}$ can further increase the sensor optimal output. While increasing the width (rw) of the resistor from $2 \mu \mathrm{m}$ (standard type) to $3 \mu \mathrm{m}$ will drop $10 \%$ of the sensor optimal output level. Increasing the diaphragm thickness (h) to $3 \mu \mathrm{m}$ will greatly reduce the sensor output. Moreover, increasing the turns of the resistor to 3 turns will increase the sensitivity as observed in Fig. 9.

\section{TESTING RESULTS AND DISCUSSIONS}

The fabricated pressure sensors have been tested by applying 100 Psi full scale pressure in steps. The measured output results are shown in Fig. 10. The input driving voltage for the sensor is 5 Volts and about -25 $\mathrm{mVolt}$ zero pressure offset and $75 \mathrm{mVolt}$ full scale output was measured. Figure 11 shows the linearity error of each measurement steps. The maximum error appears at 20 Psi and the reading is about $-0.2 \%$ FSS and the total linearity error can be observed as $\pm 0.1 \%$ FSS.

There are several ways to increase the sensitivity of the prototype pressure sensors. First, a full Wheatstone

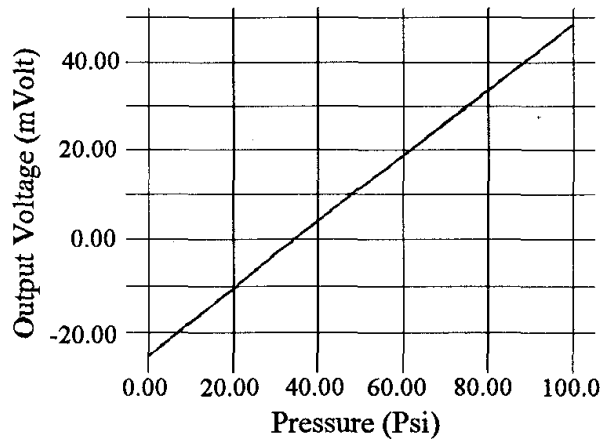

Fig. 10 Testing result from a $100 \mu \mathrm{m}$ diagram with a 3 turn sensing resistor. The sensitivity is 0.15 $\mathrm{mV} / \mathrm{V} / \mathrm{Psi}$

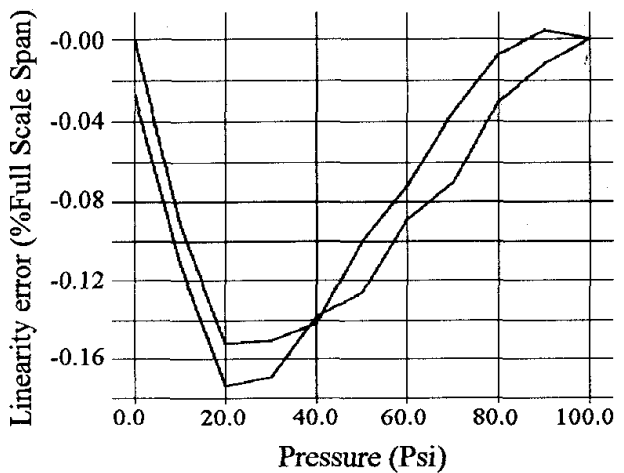

Fig. 11 Pressure vs. linearity error. The linearity error is less than $\pm 0.1 \%$ FFS. bridge can be implemented by putting the two reference resistors to optimal positions in the compressive strain region. Second, the resistance due to the non-effective portion of the resistor can be further reduced if a second high dose implantation is added in the process [1]. Third, if a better lithography equipment is used, the noneffective resistor length can be further reduced. All these steps increase the sensitivity theoretically. Finally, the linearity error measured in Fig. 11 is negative but it is positive for most other pressure sensors [4]. The cause of this phenomenon should be further investigated.

\section{CONCLUSIONS}

A newly developed fabrication process for surface micromachined diaphragm type pressure sensors has been successfully demonstrated. LPCVD polysilicon is used to hermetically seal large cavities via the aid of artificial etch channels. The design optimization for the built-in piezoresistive sensing resistors has been studied to improve both the sensitivity and linearity of the pressure sensors. Results show that the pressure sensor achieved sensitivity of $0.15 \mathrm{mV} / \mathrm{V} / \mathrm{Psi}$, linearity error of $\pm 0.1 \% \mathrm{FSS}$ and pressure hysteresis of $0.02 \% \mathrm{FSS}$.

\section{REFERENCES}

[1] H. Guckle, D.W. Burns, "Planar Processed Polysilicon Sealed Cavities for Pressure Transducers Array", 1984 IEDM, pp. 223-225, 1986.

[2] S. Sugiyama, K. Shimaoka and O. Tabata, "Surface Micromachined Micro-Diaphragm Pressure Sensors", proc. of the 6th Int. Conf. on Solid-State Sensors and Actuators Transducers '91, pp. 188-192, San Francisco, CA, USA, 1991.

[3] J.T. Kung and H.-S. Lee, "An Integrated Air-GapCapacitor Pressure Sensor and Digital Readout with Sub100 Attofarad Resolution", Journal of Microelectromechanical Systems, Vol. 1, pp. 121-129, 1992.

[4] Motorola Pressure Sensor Device Data, Phoenix, Arizona, 85036.

[5] O.N. Tufte, P.W. Chapman, and D. Long, "Silicon diffused-element piezoresistive diaphragms", J. Appl. Phys., vol. 33, pp.3322, 1962.

[6] S.K. Clark and K.D. Wise, "Pressure Sensitivity in Anisotropically Etched Thin-Diaphragm Pressure Sensors", IEEE Trans. Electron Devices, Vol. ED-26, pp. 1887-1979, Dec. 1979.

[7] Liwei Lin, K.M. McNair, R.T. Howe and A.P. Pisano, "Vacuum Encapsulated Lateral Microresonators", Proc. of the 7th Int. Conf. on Solid-State Sensors and Actuators Transducers '93, pp. 270-273, Yokohama, Japan, 1993.

[8] S. Timoshenko and S. Woinowsky-Krieger, "Theory of Plates and Shells", 2nd. ed., Chap. 6 art. 44, McGraw-Hill, New York, 1959.

[9] Swanson Analysis Systems, Inc., "ANSYS Finite Element Analysis Program", Johnson Road, P.O. Box 65, Houston, PA, 15342-0065, USA. 